\title{
REVISÃO BIBLIOGRÁFICA: O ESTADO DA ARTE DOS BENEFÍCIOS DA METODOLOGIA SEIS SIGMA NAS EMPRESAS
}

\section{Fabio Batista da Silva', José Salvador da Motta Reis ${ }^{2}$, José Glênio Medeiros de Barros $^{1}$, Antônio Henriques de Araújo Junior ${ }^{2}$, Nilo Antonio de Souza Sampaio ${ }^{1}$}

${ }^{1}$ Universidade do Estado do Rio de Janeiro / Faculdade de Tecnologia, Rod. Pres. Dutra, km 298 Bairro 27537-000 - Resende-RJ, Brasil, eng.fabiobsilva@gmail.com, glenio.barros@gmail.com, nilo.samp@terra.com.br.

${ }^{2}$ Universidade Estadual Paulista /Faculdade de Engenharia de Guaratinguetá, Avenida Dr. Ariberto Pereira da Cunha, 333, Bairro - 12244-000 - Guaratinguetá-SP, Brasil, jmottareis@gmail.com.

Resumo - A busca pela competitividade no mercado globalizado tem feito com que as organizações busquem novas maneira para se manterem competitivas e essa busca passa pela gestão de qualidade. A metodologia Seis Sigma é um programa empregado nas organizações com o intuito de potencializar os resultados empregados pelos sistemas de qualidade. A metodologia Seis Sigma contribui para o alcance de resultados sobre o problema e como consequência, tornou-se parte do Sistema de Qualidade para as ações de melhoria contínua da empresa objeto de estudo. Diante de todos os benefícios que metodologia Seis Sigma proporciona a programas de qualidade, busca-se identificar os o estado da arte na implementação da metodologia Six Sigma: Quais os pontos fundamentais da metodologia Seis Sigma. Para essa resolução, o presente trabalho tem como objetivo identificar os pilares da metodologia, para melhorar a qualidade de seus serviços e produtos.

Palavras-chave: Gestão da Qualidade, DMAIC, Zero Defeito, Garantia de Qualidade. Área do Conhecimento: Engenharias. Engenharia de Produção.

\section{Introdução}

A busca pela competitividade no mercado globalizado tem feito com que as organizações busquem novas maneira para se manterem competitivas e essa busca passa pela gestão de qualidade (LINDSTRÖM et al., 2020). As estratégias utilizadas são aquelas que oferecem o reconhecimento pelo desempenho e qualidade de seus produtos e serviços no que se refere a opinião do cliente-consumidor (GUPTA; ACHARYA; PATWARDHAN, 2012). O sucesso das organizações está na verificação de seus métodos produtivos e os processos empregados na colocação do produto e/ou serviço no mercado. Um ponto crucial na metodologia Seis Sigma é o estabelecimento de padronização para os processos produtivos (GAIKWAD; SUNNAPWAR, 2020).

A metodologia Seis Sigma é um programa empregado nas organizações com o intuito de potencializar os resultados empregados pelos sistemas de qualidade. A evolução do conhecimento faz com que cada vez mais se desenvolvam metodologias que aprimorem os produtos gerados, tanto bens como serviços. Dentro destas metodologias, o Seis Sigma conseguiu não só reunir as ferramentas da qualidade mais úteis desenvolvidas até então mas, também, reuni-las de um modo inédito fazendo com que sua aplicação seja extremamente lógica e eficaz (GUPTA; ACHARYA; PATWARDHAN, 2012).

Diante de todos os benefícios que a metodologia Seis Sigma proporciona a programas de qualidade, busca-se identificar os benefícios da implementação da metodologia Six Sigma: Quais os pontos fundamentais da metodologia Seis Sigma. Para essa resolução, o presente trabalho tem como objetivo estado da arte na implementação da metodologia, para melhorar a qualidade de seus serviços e produtos. Este trabalho está organizado em sete seções, composto por: introdução, metodologia, resultados, discussões, conclusão, agradecimentos e referências.

\section{Metodologia}


A pesquisa pode ser classificada como básica e de abordagem exploratória e qualitativa. Como procedimentos técnicos adotou-se respectivamente a pesquisa bibliográfica e análise de conteúdo (KOTHARI; GARG, 2019).

Primeiramente foram selecionados na pesquisa somente artigos publicados em inglês, por ser a língua mais comumente usada na ciência (NUNHES; OLIVEIRA, 2018). Na segunda etapa realizouse a pesquisa bibliográfica com artigos da base de dados Scopus Após a pesquisa de artigos referente a temática supracitada (KOTHARI; GARG, 2019). Por último realizou-se a análise de conteúdo que permite identificar as principais definições existentes na literatura (HSIEH; SHANNON, 2005).

\section{Resultados}

Sigma é uma letra grega utilizada pela estatística para medir o desvio-padrão (medida mais comum da dispersão estatística) de uma população. Em qualidade, o sigma é uma medida de variabilidade usada para indicar quanto dos dados insere-se nos requisitos do cliente. Quanto maior o sigma do processo, melhores os produtos ou serviços ou menor o número de defeitos (WEI; CHENG, 2020).

A Estratégia Seis Sigma busca a melhoria da empresa, a redução de erros (não conformidades dos produtos e/ou serviços diante das especificações), ou seja, a maior qualidade possível em cima dos produtos e/ou serviços oferecidos. A principal prioridade envolve a obtenção de resultados de forma planejada e clara, tanto de qualidade como financeiros (ALOSANI, 2020; GUPTA; ACHARYA; PATWARDHAN, 2012).

A utilização desta estratégia as empresas de qualquer ramo podem aumentar significativamente a probabilidade de se manterem bem sucedidas nesse novo cenário. Sendo assim, o Seis Sigma passa a ser visto não mais como um modismo no mundo dos negócios, mas ao contrário, um sistema flexível para alcançar a liderança e um melhor desempenho de negócios. O Seis Sigma se concentra na diminuição, ou até mesmo eliminação da incidência de erros, defeitos e falhas em um processo. Consiste na aplicação de métodos estatísticos a processos empresariais, orientada pela meta de eliminar defeitos (GAIKWAD; SUNNAPWAR, 2020; HAKTANIR; KAHRAMAN, 2021).

Seis Sigma é uma metodologia rigorosa que utiliza ferramentas e métodos estatísticos para DEFINIR os problemas e situações a melhorar, MEDIR para obter as informações de dados, ANALISAR a informação coletada, INCORPORAR e empreender melhorias nos processos e, finalmente CONTROLAR os processos ou produtos existentes, com a finalidade de alcançar etapas ótimas, o que por sua vez gerará um ciclo de melhoria contínua (SONY et al., 2020).

Um dos elementos da Infraestrutura do Seis Sigma é a constituição de equipes para executar projetos que contribuam fortemente para 0 alcance das metas estratégicas da empresa. $O$ desenvolvimento desses projetos é realizado com base em um método denominado DMAIC.

O método DMAIC é constituído de 5 partes: D, Define (Definir): Definir com precisão o escopo do projeto; M, Measure (Medir): Determinar a localização ou foco do problema; A, Analyse (Analisar): Determinar as causas de cada problema prioritário; I, Improve (Melhorar): Propor, avaliar e implementar soluções para cada problema prioritário; C, Control (Controlar): Garantir que o alcance da meta seja mantido a longo prazo (ALOSANI, 2020; COSTA et al., 2020; SONY et al., 2020).

A etapa D, Define (Definir): será feita a definição do cronograma preliminar do projeto e serão respondidas as seguintes perguntas: Qual é o problema; Qual a meta a ser atingida; Quais são os clientes/consumidores afetados pelo problema; Qual é o processo relacionado ao problema; Qual é o impacto econômico do projeto. Durante a execução deverá ser utilizada outra ferramenta muito útil: o mapa de raciocínio, que é importante para identificar os principais clientes/consumidores do projeto e incorporar informações geradas por procedimentos utilizados para avaliar as necessidades desses clientes (ALOSANI, 2020; COSTA et al., 2020; SONY et al., 2020).

A etapa M, Measure (Medir): deverá ser refinado ou focalizado. Duas questões devem ser respondidas: Que resultados devem ser medidos para obtenção de dados úteis focalização do problema; Quais são os focos prioritários do problema; É impossível realizar a estratificação do problema. Depois deve ser iniciado o plano de coleta de dados e deverão ser construídas Folhas de Verificação.

Com base nos dados obtidos e na forma de estratificação previamente definida, a equipe deverá analisar o impacto das várias partes do problema e identificar os problemas prioritários por meio da construção de Diagramas de Pareto (SONY et al., 2020). 
A etapa A, Analyze (Analisar): nesta etapa é importante destacar um questionamento: Por que o problema prioritário existe. As ferramentas utilizadas no Process Door que é o exame do processo gerador do problema prioritário são: Fluxograma, Mapa de Processo, Mapa de Produto, Analise do Tempo de Ciclo FMEA e FTA (ALOSANI, 2020; COSTA et al., 2020; SONY et al., 2020).

Depois vem o Data Door onde são analisados os dados provenientes do processo e suas principais ferramentas são: Avaliação de Sistemas de Medição e Inspeção, Histograma, Boxplot, Estratificação, Diagrama de Dispersão e Cartas "Multi-Vari". Deve ser realizado um brainstorming para levantar informações por meio do Diagrama de Causa e Efeito, Diagrama de Afinidades e Diagrama de relações.

No final dessa etapa as causas fundamentais do problema prioritário devem estar identificadas de modo a constituírem a base para a geração dissoluções que ocorrerá na próxima etapa do DMAIC (GUPTA; ACHARYA; PATWARDHAN, 2012; HAKTANIR; KAHRAMAN, 2021).

A etapa I - Improve (Melhorar): nesta etapa os questionamentos são os seguintes: Quais são as ideias sobre as formas para eliminação das causas fundamentais; Todas essas ideias podem ser transformadas em soluções de elevado potencial para implementação; Que soluções possivelmente levarão ao alcance da meta com menor custo é maior facilidade de execução; Como testar as soluções escolhidas com o objetivo de se garantirem o alcance da meta e a ausência de efeitos correlatos indesejáveis (ALOSANI, 2020; COSTA et al., 2020; SONY et al., 2020).

As ideias levantadas nesta fase devem ser refinadas e combinadas para darem origem às soluções potencias para alcance da meta prioritária.Com a ajuda de uma das ferramentas como Diagrama de Causa e Efeito, Diagrama de Afinidades ou Diagrama de relações. Depois deve ser utilizado o Diagrama de matriz ou Matriz de Priorização a seguir os riscos devem ser avaliados e minimizados. Depois consiste no testes na Operação das soluções prioritárias escolhidas podem ser utilizadas as ferramentas Testes de mercado e simulação (GUPTA; ACHARYA; PATWARDHAN, 2012; HAKTANIR; KAHRAMAN, 2021; SONY et al., 2020).

Após os possíveis ajustes o resultado sendo favorável será feita uma elaboração e execução de um plano para a implementação das soluções em larga escala. Caso seja desfavorável a equipe deverá retornar a etapa $\mathrm{M}$ do DMAIC. A etapa C, Control (Controlar): esta etapa consiste na avaliação do alcance da meta em larga escala. Com esse objetivo os resultados obtidos após a ampla implementação das soluções devem ser monitorados para a confirmação do alcance do sucesso. As ferramentas utilizadas são: Avaliação de Sistemas de Medição e Inspeção, Diagrama de Pareto, Carta de Controle, Histograma, Índices de Capacidade e Métricas do Seis Sigma. Se o resultado for desfavorável, a equipe retorna a etapa M (ALOSANI, 2020; COSTA et al., 2020; SONY et al., 2020)..

Se for favorável, a próxima fase consistirá na padronização das alterações realizadas no processo em consequência das soluções adotadas. Novos procedimentos operacionais padrão devem ser estabelecidos ou os procedimentos antigos devem ser revisados. Esses procedimentos devem incorporar mecanismos que garantam a realização de atividades a prova de erro (Mistake-Proofing ou Poka-Yoke) de modo a enfatizar a detecção de erros (SONY et al., 2020).

Depois deve-se definir e implementar um plano para monitoramento da performance do processo e do alcance da meta. Finalmente todas as atividades realizadas devem ser recapituladas para que seja feita uma reflexão sobre a forma de condução do projeto e também para que sejam levantados os pontos não abordados no trabalho (GUPTA; ACHARYA; PATWARDHAN, 2012).

\section{Discussões}

O principal benefício do programa Seis Sigma é minimizar custos, por meio da redução ou da eliminação de atividades que não agregam valor ao processo e da maximização da qualidade, para obter lucro em níveis ótimos. Em segundo lugar, implementar o Seis Sigma em uma organização cria uma cultura interna de indivíduos educados em um programa padronizado de caracterização, otimização e controle de processos, definindo-se processo como a atividade repetitiva envolvida no fornecimento de um serviço ou na confecção de um produto (GUPTA; ACHARYA; PATWARDHAN, 2012).

A otimização de processos faz com que eles sejam simplificados, reduzindo o número de passos e tornando-os mais rápidos e eficientes. Ao mesmo tempo, esses processos são otimizados para que não gerem defeitos nem apresentem oportunidades de erros. A eliminação de defeitos gera duas consequências(SANDNER et al., 2020; SONY et al., 2020). 
Primeiro, diminui custos, porque defeitos tornam os produtos e serviços mais caros - e quanto mais caros eles forem, menos provável será a possibilidade ou vontade das pessoas de comprá-los. Em segundo lugar, defeitos, erros e falhas desapontam os clientes, e clientela insatisfeita devolve os produtos ou não compra mais serviços. Quanto maior o número de clientes insatisfeitos com produtos e serviços, maior a tendência de se perder espaço no mercado. Ao se perder parte do mercado, perde-se também parte da receita bruta (COSTA et al., 2020; SANDNER et al., 2020).

O Seis Sigma pode ser aplicado a todos os processos e atividades econômicas. Nos processos administrativos, em especial na área de prestação de serviços e transações comerciais, existem determinadas etapas que são intangíveis. Portanto, entendê-los melhor e determinar suas características, promover a otimização, o controle e a eliminação de falhas e erros são benefícios possíveis com a implementação de um programa Seis Sigma (FITRIANA; SARAGIH; LARASATI, 2020; SONY et al., 2020).

Por exemplo, gerar um orçamento é um processo administrativo, vender um produto por telefone é um processo de serviço, assim como fazer um financiamento imobiliário é um processo de transação comercial. Determinados processos administrativos podem ter um número excessivo de etapas, causando, eventualmente, variações em seu resultado final. Daí a vantagem de aplicar o Seis Sigma visando diminuir ou eliminar a variação dos processos (FITRIANA; SARAGIH; LARASATI, 2020; WEI; CHENG, 2020).

Contudo, para alcançar e manter os benefícios do programa Seis Sigma em processos administrativos, a metodologia DMAIC deve estar estruturada de modo a assegurar a manutenção das conquistas obtidas, pois ela permite alcançar melhorias de performance elevada. Nos processos administrativos, é possível conseguir os benefícios a seguir com a aplicação da metodologia. Na fase Definir (Define), são identificados os projetos que se enquadram no programa Seis Sigma e que serão desenvolvidos na empresa com o objetivo de satisfazer as expectativas dos clientes em termos de qualidade, preço e prazo de entrega (ALOSANI, 2020; COSTA et al., 2020; SONY et al., 2020).

A habilidade da organização em atender a essa expectativa está intimamente ligada à variação de seus processos, sejam eles processos produtivos, administrativos ou transacionais, como os de serviços e de vendas. A variação de processos tem um impacto direto nos resultados financeiros da empresa, em termos de custo, tempo de ciclo e número de defeitos, de falhas e de erros que afetam a satisfação do cliente. A identificação de projetos aos quais se aplica o Seis Sigma permite à organização reconhecer como os seus processos afetam a lucratividade e definir quais processos são críticos para o negócio da empresa (STANIVUK et al., 2020; WEI; CHENG, 2020).

A fase Medir (Measure) abrange ações relacionadas à mensuração do desempenho de processos e à quantificação da variabilidade dos mesmos. Por meio de consenso entre os integrantes da equipe que trabalha no programa Seis Sigma da empresa, são identificadas as Variáveis de Entrada de Processos-Chave (KPIVs) e as Variáveis de Saída de Processos-Chave (KPOVs). Nessa fase, são utilizadas ferramentas básicas, como as métricas Seis Sigma, a Análise de Sistemas de Medição (MSA), a Análise de Modos de Falha e Efeitos (FMEA) e o Desdobramento da Função Qualidade (QFD) (BOANGMANALU et al., 2020; GUPTA; ACHARYA; PATWARDHAN, 2012; SONY et al., 2020).

$\mathrm{Na}$ fase Analisar (Analyse), são analisados os dados levantados relativos aos processos estudados, com o objetivo principal de se conhecer as relações causais e as fontes de variabilidade e de desempenho insatisfatório desses processos, visando a sua melhoria. Nessa etapa, as ferramentas utilizadas incluem visualização de dados, testes de hipótese, análise de correlação e regressão e análise de variância (WEI; CHENG, 2020).

A fase Melhorar (Improve) consiste, fundamentalmente, no desenvolvimento de Projetos de Experimentos (DOE), com o objetivo de conhecer a fundo cada processo, por meio da mudança estrutural de níveis das operações e dos diversos fatores associados. As informações obtidas com o DOE auxiliam a identificar os ajustes necessários das variáveis-chaves para modificar e otimizar o referido processo (EL-SHARKAWY et al., 2020; GUPTA; ACHARYA; PATWARDHAN, 2012).

$\mathrm{Na}$ fase Controlar (Control), são implementados diversos mecanismos para monitorar continuamente o desempenho de cada processo. Entre as técnicas adotadas, destacam-se as cartas de controle, os planos de controle, os testes de confiabilidade e os processos à prova de erros. Por fim, a metodologia DMAIC é utilizada quando um processo existente não satisfaz as necessidades ou objetivos do cliente ou apresenta um desempenho abaixo do esperado (FITRIANA; SARAGIH; LARASATI, 2020; SONY et al., 2020). 


\section{Conclusão}

Conclui-se ao final deste trabalho que diferenciar-se positivamente da concorrência e alcançar um desempenho superior é a regra para a sobrevivência. Diante dessa situação, eliminar desperdícios, adotar tecnologias avançadas, desenvolver novos produtos, envolver os colaboradores e buscar a melhoria contínua dos processos de produção tornaram-se a base de sustentação dos negócios.

Nesse contexto, as empresas que desejam manter-se à frente da concorrência devem esforçar-se para conseguir a gestão eficaz dos seus recursos para sustentar sua posição no mercado e praticar ações que possibilitem a análise dos processos e a busca das melhorias de performance. Visando auxiliar as empresas na conquista da superioridade, o Seis Sigma concentra-se na diminuição ou eliminação da incidência de erros, defeitos e falhas em um processo.

Estratégia Seis Sigma é uma extensão dos conceitos da Qualidade Total com foco na melhoria contínua dos processos, iniciando por aqueles que atingem diretamente o cliente. A estratégia Seis Sigma não é uma proposta inovadora. Ela aproveita todas as iniciativas de qualidade que estão em andamento ou que já foram implantadas na instituição, harmonizando-as e estabelecendo metas desafiadoras de redução de desperdício. Além de uma ferramenta técnica, o padrão de qualidade Seis Sigma deve ser encarado também pelo seu lado mais filosófico, que é o desenvolvimento e refinamento de uma busca incessante pela perfeição.

\section{Agradecimentos}

Este trabalho foi financiado pela Coordenação de Aperfeiçoamento de Pessoal de Nível Superior Brasil (CAPES) - Código Financeiro 001.

\section{Referências}

ALOSANI, M. S. Case example of the use of Six Sigma and Kaizen projects in policing services. Teaching Public Administration, v. 38, n. 3, p. 333-345, 3 out. 2020.

BOANGMANALU, E. et al. Minimizing damage of product using six sigma and triz methods. IOP Conference Series: Materials Science and Engineering, v. 801, p. 012101, 3 jun. 2020.

COSTA, L. B. M. et al. The effect of Lean Six Sigma practices on food industry performance: Implications of the Sector's experience and typical characteristics. Food Control, v. 112, p. 107110, jun. 2020.

\section{EL-SHARKAWY, A. et al. Integration of Sensitivity Analysis and Design for Six Sigma (DFSS) Methodology into Transient Thermal Analysis. 14 abr. 2020Disponível em: $<$ https://www.sae.org/content/2020-01-1389/>}

FITRIANA, R.; SARAGIH, J.; LARASATI, D. P. Production quality improvement of Yamalube Bottle with Six Sigma, FMEA, and Data Mining in PT. B. IOP Conference Series: Materials Science and Engineering, v. 847, p. 012011, 28 maio 2020.

GAIKWAD, L.; SUNNAPWAR, V. An integrated Lean, Green and Six Sigma strategies. The TQM Journal, v. 32, n. 2, p. 201-225, 3 jan. 2020.

GUPTA, V.; ACHARYA, P.; PATWARDHAN, M. Monitoring quality goals through lean Six- Sigma insures competitiveness. International Journal of Productivity and Performance Management, v. 61, n. 2, p. 194-203, 13 jan. 2012.

HAKTANIR, E.; KAHRAMAN, C. Design for Six Sigma and Process Capability Using Penthagorean Fuzzy Sets. In: [s.l: s.n.]. p. 1385-1395.

HSIEH, H.-F.; SHANNON, S. E. Three Approaches to Qualitative Content Analysis. Qualitative Health Research, v. 15, n. 9, p. 1277-1288, nov. 2005.

KOTHARI, C. R.; GARG, G. Research methodology methods and techniques. $4^{\circ}$ ed. Nova Deli: 
New Age International, 2019.

LINDSTRÖM, J. et al. An Initial Model for Zero Defect Manufacturing. Applied Sciences, v. 10, n. 13, p. 4570,1 jul. 2020.

NUNHES, T. V.; OLIVEIRA, O. J. Analysis of Integrated Management Systems research: identifying core themes and trends for future studies. Total Quality Management \& Business Excellence, p. 123, 13 maio 2018.

SANDNER, K. et al. A Lean Six Sigma framework for the insurance industry: insights and lessons learned from a case study. Journal of Business Economics, v. 90, n. 5-6, p. 845-878, 13 jun. 2020.

SONY, M. et al. Key Criticisms of Six Sigma: A Systematic Literature Review. IEEE Transactions on Engineering Management, v. 67, n. 3, p. 950-962, ago. 2020.

STANIVUK, T. et al. Application of Six Sigma Model on Efficient Use of Vehicle Fleet. Symmetry, v. 12, n. 5, p. 857, 22 maio 2020.

WEI, C.-C.; CHENG, Y.-L. Six Sigma project selection using fuzzy multiple attribute decision-making method. Total Quality Management \& Business Excellence, v. 31, n. 11-12, p. 1266-1289, 17 ago. 2020. 